\title{
Distanciamento e isolamento sociais pela Covid-19 no Brasil: impactos na saúde mental
}

\section{| ${ }^{1}$ Rossano Cabral Lima |}

1 Instituto de Medicina Social, Universidade do Estado do Rio de Janeiro. Rio de Janeiro-RJ, Brasil (rossanolima1@gmail.com). ORCID: 0000-0002-8583-4535

Recebido em: 27/04/2020

Aprovado em: 28/04/2020

Revisado em: 28/04/2020

DOI: http://dx.doi.org/10.1590/S0103-73312020300214

A pandemia de coronavírus tem atravessado todo o tecido social, não poupando praticamente nenhuma área da vida coletiva ou individual, com repercussões na esfera da saúde mental. Em situaçóes de epidemia, o número de pessoas psicologicamente afetadas costuma ser maior que o de pessoas acometidas pela infecção, sendo estimado que um terço a metade da população possa apresentar consequências psicológicas e psiquiátricas caso não recebam cuidados adequados (CEPEDES 2020a; ORNELL et al., 2020). Revisão de estudos sobre situaçôes de quarentena apontou alta prevalência de efeitos psicológicos negativos, especialmente humor rebaixado e irritabilidade, ao lado de raiva, medo e insônia, muitas vezes de longa duração (BROOKS et al., 2000). Contudo, dado o caráter inédito do distanciamento e isolamento sociais simultâneos de milhôes de pessoas, o impacto da atual pandemia pode ser ainda maior, levando à hipótese de "pandemia de medo e estresse" (ORNELL et al., 2020). Apesar de os primeiros artigos e relatos começarem a surgir (disponíveis para consulta em páginas como o COVID-19 Resource Centre do The Lancet), ainda há escassez de papers avaliando, de modo metodologicamente adequado, seus efeitos psicossociais nos diferentes países e grupos sociais. ${ }^{1}$

Documentos da Organização Mundial da Saúde (OMS) e do Inter-Agency Standing Committee (IASC), fórum instituído pelas Naçóes Unidas com para lidar 
com crises humanitárias, têm abordado os diversos aspectos da pandemia que se relacionam com a saúde mental, incluindo a organizaçáo dos serviços, estratégias para grupos vulneráveis do ponto de vista físico e/ou psíquico (idosos, crianças, pessoas com doenças crônicas, doenças mentais graves ou deficiências), pressões sobre os profissionais de saúde e recomendaçôes para a população em geral, envolvendo medidas de promoção e prevenção de saúde visando mitigar os efeitos do isolamento prolongado. Guias de orientação elaborados antes da atual pandemia têm sido recuperados, como o Guia PCP - Primeiros Cuidados Psicológicos (OMS, 2015), e dois documentos da Organização Pan-Americana de Saúde (OPAS), Proteção da Saúde Mental em Situaçôes de Epidemias (OPAS, 2006) e o Guia de Intervenção Humanitária do Programa de Ação Global para Superação das Lacunas em Saúde Mental da OMS (GIH-mhGAP) (OPAS; OMS, 2020), publicado em inglês em 2015 e traduzido este ano para o português.

No Brasil, começa a circular material nacional produzido por grupos de pesquisa, associaçôes e instituiçôes ligadas a categorias profissionais, com informaçôes e recomendações sobre saúde mental no contexto da pandemia, tendo os documentos internacionais como referência. Um exemplo é a série de cartilhas lançadas pelo Centro de Estudos e Pesquisas em Emergências e Desastres em Saúde (CEPEDES/ FIOCRUZ), abrangendo desde recomendaçôes à população em geral até temas mais específicos, como o cuidado de crianças em isolamento hospitalar e a violência doméstica e familiar. As iniciativas têm explorado outros recursos da internet, como as transmissóes ao vivo, como é o caso das lives "Pandemia, isolamento social e sofrimento psíquico", da Associação Brasileira de Saúde Mental (ABRASME), e "O novo Coronavírus e nossa saúde mental", do Conexão Fiocruz Brasília², e dos podcasts "As contribuiçóes da Psicologia Hospitalar na pandemia da Covid-19" e" Coronavírus e a atuação da Psicologia nas políticas de saúde e assistência social”, do Conselho Federal de Psicologia (CFP). ${ }^{3}$

Uma peculiaridade do momento é que, pela situação de distanciamento, a modalidade de teleatendimento tem ganhado espaço, tendo sido recentemente regulamentada pelo CFP (2020), Ministério da Saúde (BRASIL, 2020a) e Conselho Federal de Medicina (CFM, 2020); orientaçóes nesse sentido foram publicadas pela Cruz Vermelha Internacional (2020) e pelo CEPEDES/Fiocruz (2020b). Universidades e faculdades, sociedades psicanalíticas ${ }^{4}$ e grupos de terapeutas voluntários têm oferecido apoio e atendimento online gratuitos à populaçáo em geral 
ou para grupos específicos, como professores, funcionários técnico-administrativos e estudantes, como é o caso dos Institutos de Psicologia da UERJ e da USP, ${ }^{5}$ ou para trabalhadores da saúde. ${ }^{6}$ O Ministério da Saúde anunciou, recentemente, a criação de programa de suporte psicológico por meio de teleconsultas a profissionais da linha de frente no combate à epidemia (BRASIL, 2020b), e o Conselho Federal de Enfermagem disponibilizou um chat para apoio em saúde mental a profissionais de enfermagem envolvidos com o manejo da COVID-19.7 O estado mental dos profissionais de saúde (e outros que estão ao seu lado, como motoristas, seguranças e trabalhadores da limpeza) é motivo de preocupação especial nos documentos, devido a fatores como a pressão, estresse e burnout ligados às longas horas de trabalho, ao manejo de casos graves e ao medo da contaminação e da morte, somados à distância da família e ao risco de ser estigmatizado ou hostilizado em sua vizinhança como potenciais transmissores do coronavírus (OMS, 2015; WHO, 2020; IASC, 2020).

Os documentos institucionais incorporam diversas prescriçôes de condutas com a finalidade de melhorar a qualidade de vida e atenuar os efeitos psicológicos do isolamento. De um modo geral, as orientaçóes se repetem, com pequenas variaçôes: evitar hábitos que possam gerar sofrimento, como a desinformação e o consumo de notícias sensacionalistas; evitar igualmente os excessos de informação, sendo sugerido filtrar as fontes e reduzir o número de vezes da procura por atualização sobre a Covid-19; fornecer aos idosos, especialmente aqueles com declínio cognitivo/ demências, informaçôes claras sobre a pandemia e orientaçôes objetivas sobre medidas de higiene; buscar reduzir o estigma e a rotulação das pessoas que foram infectadas; evitar o ócio, mas também a falta de pausas e descansos no home office; organizar uma rotina que equilibre atenção a si, ao trabalho e à família; manter atividades físicas, em ambientes protegidos ou em espaços abertos sem aglomerações; praticar atividades de relaxamento e meditação; manter contato telefônico ou online com familiares e amigos; ter iniciativas solidárias em relação a vizinhos ou outras pessoas ou famílias na comunidade, especialmente pessoas idosas morando sozinhas (IASC, 2020; LA FOLLIA et al., 2020a). Uma situação especial diz respeito a falecimentos de parentes ou amigos próximos devido a Covid-19, sendo recomendado mobilizar estratégias culturalmente sensíveis para que o luto possa ser experimentado, mesmo quando os enterros tradicionais não forem possíveis (IASC, 2020).

$\mathrm{Na}$ população infanto-juvenil, recomendaçôes adicionais envolvem a organização de uma agenda que equilibre horários de estudos e tempo para 
brincar, evitando excessos de eletrônicos e internet; a ajuda no contato com avós, outros parentes e amigos; a regulaçáo do acesso a notícias sobre a pandemia, com a oferta de informaçóes adequadas a sua faixa etária; e o acolhimento a seus medos, ajudando-os a expressarem suas emoçóes, preocupaçóes e fantasias por meios lúdicos. No caso específico dos adolescentes, se acrescenta à lista de sugestôes o manejo cuidadoso, pelos pais, de características típicas que podem se exacerbar neste período, como as condutas oposicionistas e o retraimento no quarto, junto com a frustração pela impossibilidade de encontros com os pares (OMS, 2015; IASC, 2020; LA FOLLIA et al. 2020a; UNICEF, 2020a; 2020b). Há também preocupaçóes com grupos de crianças e adolescentes com características especiais, como os autistas (SBP, 2020; LA FOLLIA et al., 2020b).

É possível perceber que as estratégias prescritas têm ênfase preventiva, no sentido de produzir ou reforçar hábitos de autocuidado tidos como saudáveis, reduzindo os riscos de adoecimento mental, além de estimular uma ética comunitária que se considera escassa na vida das grandes cidades. É necessário refletir, contudo, se as recomendaçôes, ou os próprios meios virtuais nos quais elas circulam, são adequadas a todos os territórios e classes sociais. Nas populaçôes marginalizadas, as questôes geradas pelo distanciamento e isolamento têm outros matizes. Nas favelas, a menor adesão ao "fique em casa" se liga a fatores como a distinta geografia urbana composta por vielas, becos e residências com poucos cômodos, grande aglomeração e condiçóes sanitárias inadequadas; à necessidade de continuar trabalhando para se sustentar, dada a alta taxa de informalidade; e à "naturalização" do risco de vida, efeito da habituação a circular pela comunidade mesmo em dias de tiroteios e operaçóes policiais (ABRASCO, 2020). Nesse grupo, os problemas psicológicos podem se ligar aos mesmos fatores que afetam a população em geral, mas o receio de passar fome se a epidemia levar à redução da renda e perda do emprego é marca distintiva, deixando à mostra a interconexão entre a dimensão individual e social do sofrimento.

Em relação a outro grupo socialmente vulnerável, a população em situação de rua, as recomendaçôes se baseiam na abordagem humanizada na própria rua e no acolhimento institucional para fins de quarentena e isolamento. O objetivo é evitar a internação compulsória e cuidar dos problemas de saúde mental e uso de álcool e outras drogas, privilegiando as estratégias de baixa exigência, como a redução de danos (distribuição de agulhas limpas, não compartilhamento de cachimbos, becks e outros objetos pessoais) (REDE NACIONAL DE CONSULTÓRIOS NA RUA 
E DE RUA, 2020; CEPEDES, 2020c; UNODC, 2020). Esses pontos têm estado ausentes das diretrizes do Governo Federal quanto aos usuários de álcool e outras drogas, cuja política vem privilegiando a internação compulsória ou involuntária e o financiamento de dispositivos fechados, como as comunidades terapêuticas (LIMA, 2019; NUNES et al., 2019).

As publicaçôes advertem à população que, em situaçóes de distanciamento e isolamento, algumas formas de mal-estar são comuns, como a sensação de impotência, tédio, solidão, irritabilidade, tristeza e medos diversos (de adoecer, morrer, perder os meios de subsistência, transmitir o vírus), podendo levar a alterações de apetite e sono, a conflitos familiares e a excessos no consumo de álcool ou drogas ilícitas. Os idosos, em especial aqueles com declínio cognitivo ou demências, são identificados como particularmente vulneráveis a alterações emocionais e comportamentais (CEPEDES 2020a; IASC, 2000). No caso de crianças, podem reaparecer comportamentos já superados, como urinar na cama (enurese), chupar os dedos ou demandar dormir com os pais (CEPEDES, 2020d; OMS, 2015). Além disso, em sintonia com dados de epidemias passadas, começam a surgir evidências do aumento de casos de violência familiar, geralmente dirigida às mulheres e aos filhos, associada ao aumento do tempo de convivência em casa, à sobrecarga pelas múltiplas tarefas domésticas e à existência prévia de relaçôes abusivas, ao lado da menor disponibilidade de acesso a serviços públicos e instituiçôes nas quais obter ajuda e proteção (MARQUES et al., 2020; CEPEDES, 2020e).

Ainda de acordo com os documentos, a busca por atendimento especializado em saúde/saúde mental deve ocorrer em situações nas quais o sofrimento seja muito intenso e persistente, associado a pensamentos ou conduta suicida, sintomas psicóticos ou abuso recorrente de substâncias. Se por um lado é informado que os transtornos psiquiátricos imediatos mais comuns são a depressão e as reaçóes de estresse agudo transitórias, e as mais tardias podem incluir, além da depressão e do uso prejudicial de substâncias, o transtorno do estresse pós-traumático, os transtornos de adaptação e quadros psicossomáticos; por outro lado, há a preocupação com a medicalização do mal-estar e do cuidado (CEPEDES, 2020a). Aqui habitamos a zona cinzenta situada entre a normalidade e a patologia, entre o sofrimento individual e o social.

No primeiro caso, a distinção nítida entre as "reaçôes normais frente a uma situação anormal” (CEPEDES, 2020a, p. 1) e a psicopatologia carece de critérios objetivos e quantitativos, devido ao caráter valorativo e contextual das noçôes 
do normal e do patológico (BEZERRA JR., 2006). No segundo caso, falar em sofrimento social não significa apenas ressaltar a origem social da pandemia e das estratégias de distanciamento e isolamento, mas também sublinhar as circunstâncias sociais e os sentidos oferecidos pelas culturas locais que vão mediar as experiências do próprio mal-estar e da sensibilidade pela dor do outro (WILKINSON, 2006). Além disso, as categorias clínicas com as quais a saúde mental lida podem ser descritas como categorias sociais, seja por serem validadas por saberes médicopsicológicos instituídos, por terem força performativa para serem objeto de elaboração reflexiva pelos próprios sujeitos diagnosticados, ou por estarem inscritas em sistemas de circulação de valores mais amplos, articulando a economia psíquica e a ordem social (SAFATLE, 2018).

O confinamento imposto pela Covid-19, que já foi descrito como o "maior experimento psicológico do mundo" (VAN HOOF, 2020), vem colocando à prova a capacidade humana de extrair sentido do sofrimento e desafiando indivíduos e sociedade, no Brasil e em todo o planeta, a promoverem formas de coesão que amorteçam o impacto de experiências-limite na vida mental.

\section{Referências}

ASSOCIAÇÃO BRASILEIRA DE SAÚDE COLETIVA. Coronavirus nas favelas: "É difícil falar sobre perigo quando há naturalização do risco de vida". Disponível em: <https://www. abrasco.org.br/site/outras-noticias/saude-da-populacao/coronavirus-nas-favelas-e-dificil-falarsobre-perigo-quando-ha-naturalizacao-do-risco-de-vida/46098/>. Acesso em: 24 abr. 2020.

BEZERRA JR., B. O normal e o patológico: uma discussão atual. In: SOUZA, A. N.; PITANGUI, J. Saúde, corpo e sociedade. Rio de Janeiro: Editora UFRJ, 2006. p. 91-109.

BRASIL. Ministério da Saúde. Portaria no 467, de 20 de março de 2020a. Dispóe, em caráter excepcional e temporário, sobre as açôes de Telemedicina, com o objetivo de regulamentar e operacionalizar as medidas de enfrentamento da emergência de saúde pública de importância internacional previstas no art. $3^{\circ}$ da Lei no 13.979, de 6 de fevereiro de 2020, decorrente da epidemia de COVID-19. Disponível em: <http://www.in.gov.br/en/web/dou/-/portaria-n-467de-20-de-marco-de-2020-249312996>. Acesso em: 25 abr. 2020.

. Saúde vai investir $R \$ 2,3$ milhöes em suporte psicológico a profissionais de saúde. Publicado em 23/04/2020b. Disponível em: <https://www.gov.br/pt-br/noticias/saude-e-vigilanciasanitaria/2020/04/ministerio-da-saude-vai-investir-em-suporte-psicologico-a-profissionais-desaude>. Acesso em: 25 abr. 2020. 
BROOKS, S. K., et al. The psychological impact of quarantine and how to reduce it: rapid review of the evidence. The Lancet, v. 395, n. 10227, p. 912-920, March 2020.

CENTRO DE ESTUDOS E PESQUISAS EM EMERGÊNCIAS E DESASTRES EM SAÚDE; FUNDAÇÃO OSWALDO CRUZ. Saúde mental e Atenção Psicossocial na Pandemia COVID-19: Recomendaçôes gerais. Brasília, 2020 a.

- Saúde mental e Atenção Psicossocial na Pandemia COVID-19: Recomendações aos psicólogos para o atendimento online. Brasília, 2020b.

Saúde mental e Atenção Psicossocial na Pandemia COVID-19: Recomendaçôes para os consultórios na rua e a rede de serviços que atuam junto com a população em situação de rua. Brasília, 2020c.

. Saúde mental e Atenção Psicossocial na Pandemia COVID-19. Recomendaçóes para o cuidado de crianças em situação de isolamento hospitalar. Brasília, 2020d.

. Saúde mental e Atençâo Psicossocial na Pandemia COVID-19: Violência doméstica e familiar na COVID-19. Brasília, 2020e.

CONSELHO FEDERAL DE MEDICINA. Esclarecimento do CFM sobre a lei da telemedicina. Brasília, 25 de abril de 2020. Disponível em: <http://portal.cfm.org.br/images/ PDF/notacfmhonorarioscovid19.pdf>. Acesso em: 24 abr. 2020.

CONSELHO FEDERAL DE PSICOLOGIA. Resolução no 4, de 26 de março de 2020. Dispóe sobre regulamentação de serviços psicológicos prestados por meio de Tecnologia da Informação e da Comunicação durante a pandemia do COVID-19. Disponível em: <http://portal.cfm.org.br/ images/PDF/2020_oficio_telemedicina.pdf>. Acesso em: 25 abr. 2020.

CRUZ VERMELHA INTERNACIONAL. Primeiros cuidados psicológicos, remotos, durante o surto de COVID-19. Orientação provisória, março 2020. Disponível em: <https://pscentre.org/ wp-content/uploads/2020/03/IFRC-PS-Centre-Remote-PFA-during-a-COVID-19.-InterimGuidance.-Portuguese.pdf>. Acesso em: 23 abr. 2020.

FILGUEIRAS, A.; STULTS-KOLEHMAINEN, M. The Relationship Between Behavioural and Psychosocial Factors Among Brazilians in Quarantine Due to COVID-19 (3/31/2020). Disponível em: <https://ssrn.com/abstract=3566245> ou <http://dx.doi.org/10.2139/ ssrn.3566245>. Acesso em 27 abr. 2020.

INTER-AGENCY STANDING COMMITTEE. Como lidar com os aspectos psicossociais e de saúde mental referentes ao surto de COVID-19. Versão 1.5, março de 2020. Disponível em: < https:// interagencystandingcommittee.org/system/files/2020-03/IASC\%20Interim\%20Briefing\%20 Note $\% 20$ on\%20COVID-19\%20Outbreak\%20Readiness\%20and\%20Response\%20 Operations\%20-\%20MHPSS\%20\%28Portuguese\%29.pdf>. Acesso em: 25 abr. 2020. 
LA FOLLIA. Laboratório de Pesquisa em Saúde Mental e Terapia Ocupacional da UFSCAR et al. Cuidando da sua saúde mental em tempos de Coronavirus. 2020a. Disponível em: <http://www. unifap.br/wp-content/uploads/2020/03/coronavirus_saudemental.pdf>. Acesso em: 25 abr. 2020. . Orientaçóes às famílias de crianças e adolescentes com autismo em tempos de coronavírus. 2020b. Disponível em: <http://biblioteca.cofen.gov.br/wp-content/uploads/2020/03/ Orientac\%CC $\%$ A7o\%CC $\% 83$ es-a $\%$ CC $\% 80$ s-fami $\%$ CC $\% 81$ lias-de-crianc\%CC $\%$ A7as-eadolescentes-com-autismo-em-tempos-de-coronavi\%CC\%81rus.pdf>. Acesso em: 25 abr. 2020. LIMA, R. C. O avanço da Contrarreforma Psiquiátrica no Brasil. Physis, v. 29, n. 1, e290101, 2019. Disponível em: <http://www.scielo.br/scielo.php?script=sci_arttext\&pid=S0103$73312019000100100 \& \operatorname{lng}=\mathrm{pt} \& \mathrm{nrm}=\mathrm{iso}>$.

MARQUES, E. S., et al. A violência contra mulheres, crianças e adolescentes em tempos de pandemia pela COVID-19: panorama, motivaçóes e formas de enfrentamento. Cad. Saúde Pública. Rio de Janeiro, v. 36, n. 4, e00074420, abr. 2020. Disponível em: <http://cadernos. ensp.fiocruz.br/csp/artigo/1033/a-violncia-contra-mulheres-crianas-e-adolescentes-em-temposde-pandemia-pela-covid-19-panorama-motivaes-e-formas-de-enfrentamento>.

NUNES, M. D. O. et al. Reforma e contrarreforma psiquiátrica: análise de uma crise sociopolítica e sanitária a nível nacional e regional. Ciência \& Saúde Coletiva, v. 24, n. 12, p. 4489-4498, 2019.

ORGANIZAÇÃO MUNDAL DA SAÚDE; WAR TRAUMA FOUNDATION; VISÃO GLOBAL INTERNACIONAL. Primeiros Cuidados Psicológicos: guia para trabalhadores de campo. OMS: Genebra, 2015.

ORGANIZAÇÃO PAN-AMERICANA DA SAÚDE. Proteção da saúde mental em Situaçôes de Epidemia. Tradução do original em espanhol. Organização Pan-Americana de Saúde, 2006 Disponível em: >https://www.paho.org/hq/dmdocuments/2009/Protecao-da-Saude-Mentalem-Situaciones-de-Epidemias_Portugues.pdf>. Acesso em: 22 abr. 2020.

_; ORGANiZAÇÃO MUNDIAL DE SAÚdE. Manejo Clínico de Condiçóes Mentais, Neurológicas e por Uso de Substâncias em Emergências Humanitárias. Guia de Intervenção Humanitária mhGAP. Brasília: Organização Pan-Americana da Saúde, 2020.

ORNELL, F. et al. "Pandemic fear" and COVID-19: mental health burden and strategies. Braz. J. Psychiatry, São Paulo, 2020. Disponível em <http://www.scielo.br/scielo.php?script=sci_ arttext\&pid=S1516-44462020005008201\&lng=en\&nrm=iso>.

REDE NACIONAL DE CONSULTÓRIOS NA RUA OU DE RUA. O cuidado em saúde da população em situação de rua na pandemia do COVID-19. 2020. Disponível em <https://www. facebook.com/groups/1666581633593990/>. 
SAFATLE, V. Em direção a um novo modelo de crítica: as possibilidades de recuperação contemporânea do conceito de patologia social. In: SAFATLE, V.; SILVA JÚNIOR, N.; DUNKER, C. Patologias do social: arqueologias do sofrimento psíquico. São Paulo: Autêntica, 2018, p. 7-31.

SOCIEDADE BRASILEIRA DE PEDIATRIA; DEPARTAMENTO CIENTÍFICO DE NEUROLOGIA. COVID-19 e Transtorno do Espectro Autista. 2020. Disponível em: <https:// www.sbp.com.br/fileadmin/user_upload/22455c-NA_-_COVID-19_e_Transtorno_do_ Espectro_Autista__1_.pdf>. Acesso em: 22 abr. 2020.

FUNDO DAS NAÇÓES UNIDAS PARA A INFÂNCIA. Como falar com crianças sobre o coronavirus. Brasília: Unicef, 2020a. Disponível em: <https://www.unicef.org/brazil/comofalar-com-criancas-sobre-coronavirus>. Acesso em: 23 abr. 2020.

. Como adolescentes podem proteger sua saúde mental durante o surto do coronavirus.

Brasília: Unicef, 2020b. Disponível em: <https://www.unicef.org/brazil/historias/comoadolescentes-podem-proteger-sua-saude-mental-durante-o-surto-de-coronavirus $>$. Acesso em: 21 abr. 2020.

VAN HOOF, E. Lockdown is the world's biggest psychological experiment - and we will pay the price. Disponível em: <https:/www.weforum.org/agenda/2020/04/this-is-the-psychologicalside-of-the-covid-19-pandemic-that-were-ignoring/>. Acesso em: 25 abr. 2020.

WILKINSON, I. Health, risk and "social suffering”. Health, Risk \& Society, v. 8, n. 1, p. 1-8, March 2006.

WORLD HEALTH ORGANITATION. Mental health and psychosocial considerations during the COVID-19 outbreak. 18 March 2020. Disponível em: <https://www.who.int/publicationsdetail/mental-health-and-psychosocial-considerations-during-the-covid-19-outbreak>. Acesso em: 24 abr. 2020 .

\section{Notas}

${ }^{1}$ A produção científica sobre esse tema, especialmente sob a forma de preprints, tem sido acelerada. Enquanto este texto estava sendo finalizado, foi publicado estudo realizado no Brasil por pesquisadores da UERJ e da Yale University apontando aumento de sintomas de ansiedade, depressão e estresse durante a pandemia, com maiores taxas em mulheres, em pessoas vivendo com idosos em casa, naquelas mantendo o trabalho fora, de menor escolaridade e mais jovens (FILGUEIRAS, A.; STULTS-KOLEHMAINEN, 2020).

${ }^{2} \mathrm{O}$ conteúdo da transmissão pode ser acessado em: <https://www.fiocruzbrasilia.fiocruz.br/conexao-fiocruz-brasilia-aborda-os-cuidados-da-saude-mental-em-meio-a-pandemia-do-novo-coronavirus/>.

${ }^{3} \mathrm{O}$ podcast pode ser acessado em: < https://site.cfp.org.br/coronavirus/1-inicio/>.

${ }^{4}$ Como exemplos, citamos as iniciativas da Sociedade de Psicanálise do Rio de Janeiro (http://sprj.org. br/site/solidaria/); da Sociedade Psicanalítica de Porto Alegre (https://sppa.org.br/noticias/formulario-atendimento-solidario); e do Círculo Psicanalítico do Rio de Janeiro (http://cprj.com.br/). 
5 No caso do IP-UERJ, o formulário pode ser acessado em: < https://drive.google.com/open?id=1i-L3jDAd-oYoMXIZFXo9bYO7cXSLGVlgfRUmetB5UHQ>. No caso da USP, pode ser acessado em: $<$ http://www.ip.usp.br/site/covid-19-apoio-psicologico-online/>.

${ }^{6}$ Como é o caso da Sociedade Brasileira de Psicanálise de São Paulo (http://sbpsp.org.br/atividades/ eventos-sbpsp/item/1725-escuta-psicanalitica-para-profissionais-de-saud.html) e da Faculdade de Medicina da UFMG (https://www.medicina.ufmg.br/telepansaude/).

${ }^{7} \mathrm{O}$ chat pode ser acessado em: < http://www.juntoscontracoronavirus.com.br/>. 